\title{
Immunomodulatory activity of polyphenols derived from Cassia auriculata flowers in aged rats.
}

\begin{abstract}
The immunomodulatory activity of Cassia auriculata (CA)-derived polyphenols was tested on aged rats. Rats (24-26 months old) were given CA polyphenols supplementation at doses of 25,50 , and $100 \mathrm{mg} / \mathrm{kg}$ for 28 days. Flow cytometry analysis of CA polyphenols-treated aged rats showed increased $\mathrm{T}$ and $\mathrm{B}$ cells percentage along with enhanced proliferation of splenocytes in both resting and LPS-stimulated cells. Increased percentage of pan T cells is further supported by an elevation of CD4+, CD8+, and CD4+CD25+ regulatory cells. In terms of innate immune cell activity, CA polyphenol supplementation reduced the oxidative burst activity of neutrophils in response to PMA and Escherichia coli activation. Our results collectively show that polyphenols derived from CA boost $\mathrm{T}$ cell immunity by increasing the number of $\mathrm{T}$ cells and its sensitivity towards stimulants and decreasing ROS production by neutrophils that could potentially harm multiple biological systems in aged individuals.
\end{abstract}

Keyword: Cassia auriculata; Immunosenescence; T cells; Neutrophils; Splenocytes. 